\title{
A Case of Allergic Dermatitis after Self-Treatment with Propolis: Case Report
}

\author{
Monika Fida ${ }^{1}$, Suela Kellici ${ }^{2^{*}}$, Mehmet Hoxha ${ }^{3}$, Etleva Qirko ${ }^{3}$ \\ ${ }^{1}$ Faculty of Medicine - Dermatology, Tirana, Albania; ${ }^{2}$ Faculty of Medicine, Pharmaceutical Department, Tirana, Albania; \\ ${ }^{3}$ Faculty of Medicine - Allergology, Tirana, Albania
}

\begin{abstract}
Citation: Fida M, Kellici S, Hoxha M, Qirko E. A Case of Allergic Dermatitis after Self-Treatment with Propolis: Case Report. OA Maced J Med Sci. $2013 \quad$ Dec 15; 1(1):87-89. http://dx.doi.org/10.3889/oamjms.2013.017 Key words: Allergic contact dermatitis; propolis; vesiculo-bullous elements; eczematous elements; medical advice.

"Correspondence: Prof. Suela Kellici. Faculty of Pharmacy, Pharmaceutical Department Tirana, Albania. Phone: 00355 672075041. EMail: skellici@gmail.com

Received: 18-Sep-2013; Revised: 23-Oct2013; Accepted: 24-Oct-2013; Online first: 05-Nov-2013

Copyright: ๑ 2013 Fida M. This is an openaccess article distributed under the terms of the Creative Commons Attribution License, which permits unrestricted use, distribution, and reproduction in any medium, provided the original author and source are credited.

Competing Interests: The authors have declared that no competing interests exist.
\end{abstract}

\begin{abstract}
Background: Propolis or bee-glue is used as adjuvant in the therapy because of its antiseptic anti-inflammatory, anesthetic and antibacterial properties. Propolis is found in a number of products such as cosmetics, shampoos, toothpastes, lip balms, creams and ointments. In dermatology propolis has been used for wound healing, treatment of ulcers and eczema, and regeneration of the skin. The sensitizing properties of propolis have been reported in the literature.

Aim: The aim of this study was to present a case of allergic contact dermatitis after self-treatment with propolis.

Methods: We report a case of 46 years old man who presented severe vesiculo-bullous elements on the leg after the treatment with bee-glue for the eczematous elements.

Results: The diagnosis of allergic contact dermatitis to propolis was confirmed due to patch tests.

Conclusions: Here we show that allergic contact dermatitis from propolis should be taken in consideration if an allergic reaction is evident. Dermatologists and general physicians should be aware of propolis as an important allergen that can cause Allergic Contact Dermatitis. The therapeutic use of propolis should be performed under medical advisory.
\end{abstract}

\section{Introduction}

Propolis or bee-glue is used as adjuvant in the therapy because of its antiseptic, antiinflammatory, anesthetic and antibacterial properties. $[1,2]$. It is the cause of an increasing number of cases of allergic contact dermatitis (ACD). Propolis is found in a number of products such as cosmetics, shampoos, toothpastes, lip balms, creams and ointments [1-3]. In dermatology propolis has been used for wound healing, treatment of ulcers and eczema, and regeneration of the skin [1-3].

The aim of this paper is to report an acute case of ACD by propolis after selfmedication.

\section{Report}

A 46 years old veterinary man had a history with chronic eczema. He was treated ambulatory for eczema and never did the patch tests before. The patient did not have an atopic history.

He asked for an emergency visit for a vesiculo-bullous dermatitis in lower extremities. The patient refered that he was trying to treat the symptoms of leg eczema (skin elements and itching) with original bee-glue for 2 days (twice a day) because he read about its therapeutic properties in an alternative medicine book.

In the clinical examination were constated: Evident erythema, vesiculous bullous elements and edematous leg. The borders with the unaffected skin were noticed. The vesiculous-bullous elements were filled with serous liquid and in some places the necrosis was evident. The patient complained for severe itching and burning sensation.

The clinical view mimics a strong Allergic contact dermatitis or a Pemphigus vulgaris. 
Based on the history of the disease, clinical features and the localisation just to the contact with the preparation, we concluded to diagnose: Allergic Contact Dermatitis.

The treatment of the patient was started quickly with oral corticosteroids, antihistamines and topical treatments of the bullous elements (antiseptics and corticosteroid creams). After the complete recovery we performed patch tests (manufactured by Chemotechnique Diagnostics) in order to assess whether the patient was allergic to propolis or other allergens. Patch tests were performed according to the International Contact Dermatitis Research Group Guidelines.

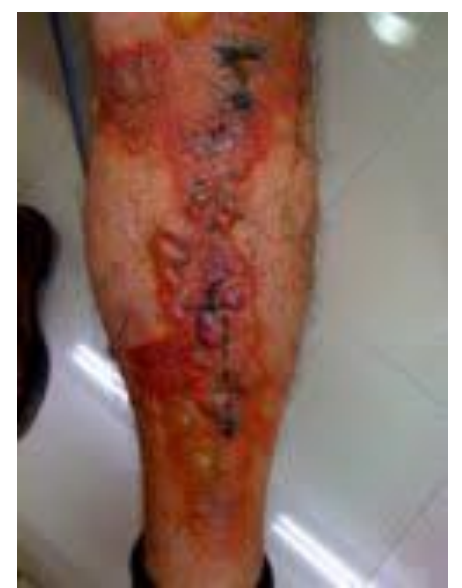

Figure1: Vesiculo bullous elements and evident necrosis.

\section{Results}

After the complete recovery of the skin and based on the guidelines we used the European Standard Series of Patch tests. The results were strongly positive reaction $(+++)$ to propolis and positive reaction $(+)$ to nickel. The reading of the results was made after 48 and 72 hours. The concentration of propolis tested was $20 \%$ in petrolatum. There was no evidence of skin irritation in this concentration. The evidented vesiculous elements induced by propolis were circumphered by erythema, while nickel induced just an erythematous reaction (the nickel concentration was $0.20 \mathrm{mg} / \mathrm{cm}^{2}$ ).

The patient was recommended to avoid the contact with products that contain propolis and other synonyms of propolis such are propolis resin, propolis wax, bee bread, bee glue, propolis balsam. No relapses of the dermatitis were observed during the period of observation ( 6 months).

\section{Discussion}

Propolis has a long history of use in alternative medicine since the Greek and Roman ancient period. Today propolis is found in a number of "natural" products, including lipbalms, cosmetics, lotions and creams, toothpaste and shampoos. Propolis is used for medicinal preparation because of its antiseptic, antinflammatory and anesthetic properties.

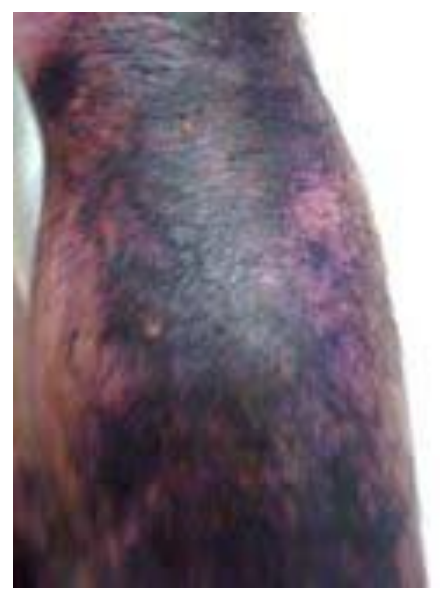

Figure 2: One week after treatment.

The sensitizing properties of propolis were reported by many authors $[1-5,10]$ According to the literature, $1.2-6.6 \%$ of patch tested patients result sensitive to propolis [2].

Propolis is part of European Standart patch test series and it is patch tested in a $10 \%$ or $20 \%$ concentration in petrolatum.

The main propolis sensitization causes are the use of cosmetics and the occupational bee keeping $[1,3]$. It is a type IV reactin of hypersensitivisation $[1,3,5,10]$. In our case, the sensitization cause was the selfmedication of leg's eczema. Similarly to our case, the sensitization to propolis after selfmedication is reported in a 35-yearold Asian woman who used the propolis ointment as medication for a minor trauma in her foot [3].

Propolis contains different allergens $[1,6,7]$. The main allergens are 3-methyl-2-butenyl caffeate and phenylethyl caffeate [6, 7]. However, propolis contains many other allergens such are benzyl cinnamate, benzyl salicilate, etc [2, 8]. A number of these compounds are part of Peruvian balsam (such as acid cinnamic esters), which is included in European Standard Series of Patch test allergens [9]. As long as the tested Peruvian balsam resulted negative, the causative allergen in our case is likely to be a propolis compound that is not present in the mentioned balsam (such as 3-methyl-2-butenyl caffeate and phenylethyl caffeate) [2, 6, 7].

As a conclusion, dermatologists and general physicians should be aware of propolis as an important allergen that can cause ACD. The therapeutic use of propolis should be performed under medical advisory. 


\section{References}

1. Hausen BM, Wollenweber E, Senff H, Post B. Propolis allergy. Origin, properties, usage and literature review. Contact Dermatitis. 1987; 17: 163-170.

2. Walgrave SE, Warshaw EM, Glesne LA. Allergic contact dermatitis from propolis. Dermatitis. 2005; 16: 209-215.

3. Ting PT, Silver S. Allergic contact dermatitis to propolis. J Drugs Dermatology. 2004; 3: 685-686.

4. Ayala F, Lembo G, Nappa P, Balato N. Contact dermatitis from propolis. Contact Dermatitis. 1985; 12: 181-182.

5. Raton JA, Aguirre A, Diaz-Perez JL. Contact dermatitis from propolis. Contact Dermatitis. 1990; 22: 183-184.

6. Hausen BM, Evers P, Stuwe HT, et al. Propolis allergy (IV). Studies with further sensitizers from propolis and constituents common to propolis, poplar buds and balsam of Peru. Contact Dermatitis. 1992; 26: 34-44.

7. Hausen BM, Wollenweber E. Propolis allergy (III) Sensitization studies with minor constituents. Contact Dermatitis. 1988; 19: 296-303.

8. Hausen BM. Evaluation of the main contact allergens in propolis (1995 to 2005). Dermatitis. 2005; 16: 127-129.

9. Schuler TM, Frosch PJ. [Propolis-incuced contact allergy] Hautarzt. 1988; 39: 139-142. [in German].

10. Susan E. Walgrave, Erin M. Warshaw, Lynn A. Glesne. Allergic contact dermatitis from Propolis. Dermatitis. 2005;16(4):209-215. 